\title{
Impact of Oxidative Stress and Newer Antiepileptic Drugs on the Albumin and Cortisol Value in Severe Motor and Intellectual Disabilities With Epilepsy
}

\author{
Masahito Morimoto $^{\mathrm{a}, \mathrm{c}}$, Toshiaki Hashimoto ${ }^{\mathrm{a}}$, Taisuke Kitaoka ${ }^{\mathrm{b}}$, Shojiro Kyotani ${ }^{\mathrm{b}}$
}

\begin{abstract}
Background: Epilepsy is a common complication in patients with severe motor and intellectual disabilities (SMID). There are no reports as yet of the effects of these medications in vivo other than their epileptic efficacy. The purpose of this study was to clarify the effects of the newer antiepileptic drugs (AEDs) on the blood biochemical parameters and oxidative stress in SMID with epilepsy by comparing the therapeutic effects between a group of patients receiving lamotrigine (LTG) and levetiracetam (LEV) in addition to the conventional AEDs (newer AED group) and a group receiving conventional AEDs alone (old AED group).
\end{abstract}

Methods: The study population consisted of 44 SMID patients with epilepsy, of which 23 were allocated to the newer AED group and 21 were allocated to the old AED group. In the newer AED group, measurements of the reactive oxygen metabolites (d-ROMs), biological antioxidant potential (BAP) and serum albumin were carried out at the following two time points: 1 week before and 1 year after the start of administration of the newer AEDs. In the old AED group, measurements of the same variables were performed at two time points 1 year apart.

Results: A significant decrease of the d-ROM levels and a significant increase of the BAP were noted in the newer AED group. A significant elevation of the serum albumin was also evident. In the old AED group, a significant increase of the d-ROMs levels was noted at the second measurement. Cortisol levels which have been described to be related to the albumin, revealed a significant decrease of the serum cortisol in relation to elevation of serum albumin in the newer AED group.

Conclusions: The present study results suggest that the addition of

Manuscript submitted December 4, 2017, accepted December 20, 2017

aJapanese Red Cross Tokushima Hinomine Rehabilitation Center for People with Disabilities, Tokushima, Japan

${ }^{\mathrm{b}}$ Tokushima Bunri University, Graduate School of Pharmaceutical Sciences, Tokushima, Japan

${ }^{\mathrm{c} C o r r e s p o n d i n g ~ A u t h o r: ~ M a s a h i t o ~ M o r i m o t o, ~ J a p a n e s e ~ R e d ~ C r o s s ~ T o k u s h i m a ~}$ Hinomine Rehabilitation Center for People with Disabilities, Chuden-cho, Komatsushima-shi, Tokushima 773-0015, Japan.

Email: morimoto@hinomine-mrc.jp

doi: https://doi.org/10.14740/jocmr3289w newer AEDs reduces the oxidative stress load and improves the antioxidant potential of the body. Furthermore, the present data also demonstrate that the newer AEDs have indirect impact on biological parameters.

Keywords: Severe motor and intellectual disabilities; Oxidative stress; Epilepsy; Albumin; Cortisol

\section{Introduction}

Oxidative stress is a state where the balance between the oxidative reactions and the biological antioxidant potential is upset by excessive production of reactive oxygen species (ROS) and free radicals, resulting in an excessively oxidized state exerting harmful effects in vivo [1]. It has also been demonstrated in recent years that such enhanced oxidative stress brought about by excessive production of ROS and free radicals results not only in the acceleration of aging, but also various other diverse disorders [2].

Our previous study in which we measured the serum levels of d-ROMs and BAP as markers of the degree of oxidative stress confirmed an association between epilepsy in patients with SMID and the oxidative stress level [3]. Patients with SMID are practically incapable of self-expression because of severe intellectual disability combined with severe physical handicaps; therefore, it is difficult to gain subjective information from these patients. Hence, control of epilepsy is even more difficult in these patients, with a consequent increase in the intractability of the epilepsy giving rise to elevation of the oxidative stress level.

Sudden death is recognized as a common cause of death in subjects with SMID [4, 5], which underscores the need for prevention/early detection of any in the everyday lives of these patients. In view of the difficulty in communicating with these patients, identification of objective indicators useful for screening and treatment of a variety of disorders and prediction of the clinical condition is of great importance.

Useful objective indicators would be those whose measurement would not prove to be cumbersome in routine clinical settings. Therefore, we identified the serum level of albumin as a known biological antioxidant among the currently extensively measured blood biochemical parameters. Albumin plays the role of an antioxidant as it becomes oxidized by ROS such as free radicals [6]. The purpose of this study was to determine 
the effects of AEDs used for the treatment of intractable epilepsy in SMID patients on the serum levels of albumin, and to clarify how albumin, an oxidant in vivo, might affect the oxidative stress levels and other bio-factors in SMID patients with epilepsy.

\section{Materials and Methods}

\section{Subjects and drugs}

The study population consisted of 44 SMID patients with epilepsy who were continually hospitalized in the Japanese Red Cross Tokushima Hinomine Rehabilitation Center for People with Disabilities between January 1, 2013, and August 31, 2016. The patients were classified into two groups according to the type of AEDs that they received: a group receiving treatment with conventional AEDs alone (old AED group; $n=21$ ) and a group receiving treatment with the newer AEDs in addition to the conventional drugs (newer AED group; $\mathrm{n}=23$ ). "Newer AEDs" here denote the two agents, LTG and LEV, which were approved for use in Japan in December 2008 and are highly recommended worldwide [7]; "old AEDs" refer to other antiepileptic agents that were approved much earlier in time.

\section{Exclusion criteria}

Patients who were not continually hospitalized for treatment during this survey period were excluded from the study. Those who had received any drug within 6 months prior to the baseline blood sampling that could potentially affect the hematologic/blood biochemical parameter values were also excluded from the study (e.g., albumin preparations, hepatoprotective drugs, nephroprotective drugs, sodium chloride preparations, various blood transfusion products, granulocyte-colony stimulating factor (G-CSF) preparations, iron preparations, and erythropoietin preparations).

\section{Survey items}

Information on the following variables were obtained from the patients' medical records: gender, age (at the time of the survey), primary disease, complications (concurrent diseases) that could possibly affect the levels of the oxidative stress markers, number and type(s) of AEDs prescribed, and blood biochemical parameter values (serum albumin, serum aspartate aminotransferase (AST), serum alanine aminotransferase (ALT), blood urea nitrogen (BUN), serum creatinine, serum sodium, serum chloride, blood erythrocyte count, hemoglobin, hematocrit, blood leukocyte counts, and blood platelet count).

\section{Assay parameters}

The blood samplings and assays were carried out at two time points in both groups; in the newer AED group, the samplings were performed about 1 week prior to the initiation of the newer AEDs (measurement point 1) and at about 1 year after the start of administration of the newer AEDs (measurement point 2 ); in the old AED group, the samplings were conducted at two time points separated by an interval of about a year: between April and August 2015 (measurement point 1) and between April and August 2016 (measurement point 2); thus, the interval between the two samplings was approximately 1 year in both the groups. The blood samples were collected when the patients were in their usual stable state and were not in a post-ictal phase or suffering/recovering from an infection, in the morning, before they took their breakfast/morning doses of the AEDs (at $6 \mathrm{am}$ ). The blood levels of the d-ROMs as indicators of the oxidative reaction level, the BAP as a marker of the biological antioxidant potential, and the blood biochemical parameters were measured in the blood samples. The assay results are presented in Table 1.

\section{Assay methods}

\section{Biochemical parameters (albumin)}

The serum concentrations of albumin were determined with a LAboSPECT006 (HITACHI Ltd, Tokyo, Japan) using the reagent, CicaLiquid ALB (Kanto Chemical Co., Inc., Tokyo, Japan).

\section{Oxidative reaction level marker (blood levels of d-ROMs)}

Plasma levels of these markers were measured with the free radical analyzer, FREE CARRIO DUO (Diacron International srl, Grosseto, Italy). Twenty microliter of plasma was pipetted into a cuvette containing a $\mathrm{pH}$ 4.8-buffer, followed by mixing of the contents of the cuvette by inversion (to allow $\mathrm{Fe}^{2+}$ and $\mathrm{Fe}^{3+}$ separation from the plasma proteins). This was expected to lead to decomposition of the hydrogen peroxide in the plasma to alkoxyl radicals and peroxy radicals catalyzed by $\mathrm{Fe}^{2+}$ and $\mathrm{Fe}^{3+}$. Then, $20 \mu \mathrm{L}$ of a chromogen (N,N-diethylparaphenylenediamine) was pipetted to allow oxidation of the chromogen by free radicals to yield red-colored radical cations. The mixture in the cuvette was mixed by inversion of the cuvette and placed in a spectrophotometer to measure the radical cations at $505 \mathrm{~nm}$. The measured quantity was expressed in CARR units (U.CARR), where 1 U.CARR corresponded to $0.08 \mathrm{mg} / \mathrm{dL}$ of $\mathrm{H}_{2} \mathrm{O}_{2}$.

\section{Marker of the biological antioxidant potential (BAP test)}

This marker was measured with the free radical analyzer, FREE CARRIO DUO (Diacron International srl, Grosseto, Italy). Fifty microliter of a chromogenic reagent (containing $\mathrm{Fe}^{3+}$ ) for BAP was pipetted into a cuvette to allow red coloration, and the contents were mixed by inversion of the cuvette, followed by spectrophotometric measurement of 
Table 1. Measurement Evaluation Standard Value of d-ROMs Test

\begin{tabular}{|lll}
\hline Evaluation & Standard value & Unit \\
\hline d-ROMs test & & *U.CARR \\
Normal range & $250-300$ & \\
Border range & $301-320$ & \\
Low-level oxidative stress & $321-340$ & \\
Intermediate level oxidative stress & $341-400$ & \\
High-level oxidative stress & $401-500$ & \\
Very high-level oxidative stress & $>500$ \\
BAP test & & \\
Normal range & $<2,200$ & \\
Border range & $2,000-2,200$ & \\
Slight lack state & $1,800-1,999$ \\
Lack state & $1,600-1,799$ & \\
Severe lack state & $1,400-1,599$ & \\
Very severe lack state & $<1,400$ & \\
\hline *1 U.CARR = 0.08 mg/100 $\mathrm{mL} \mathrm{H}_{2} \mathrm{O}_{2}$. & &
\end{tabular}

the coloration. Subsequently, $10 \mu \mathrm{L}$ of plasma was added to the cuvette and mixed. The cuvette was then placed in a thermostat to allow reaction in the mixture for $5 \mathrm{~min}$. Finally, the cuvette was placed in a spectrophotometer for measurements at $505 \mathrm{~nm}$. The assay results were expressed in $\mu \mathrm{mol} / \mathrm{L}$.

\section{Measurement of the plasma cortisol level}

The plasma (heparinized) level of cortisol in the samples was quantified by electrochemiluminescence immunoassay (ECLIA) using the Blood Test Cortisol Kit ECLusys ${ }^{\circledR}$ reagent.

\section{Statistical analysis}

The statistical significance level was set at a two-sided 5\%, where $\mathrm{P}<0.05$ was considered as indicative of statistical significance. Regarding the relationship between albumin and cortisol, the relationship between both items was analyzed using Pearson's $r$ and a correlation line. In the newer AED group and the old AED group, comparison of blood test values between measurement points 1 and 2 was analyzed using the Wilcoxon signed-rank test.

\section{Ethical considerations}

This study was conducted with the approval of the Ethical Committee of the Japanese Red Cross Tokushima Hinomine Rehabilitation Center for People with Disabilities. Written informed consent for participation in the study was obtained from a family member of each study subject, since SMID patients themselves have difficulty in expressing their intention either verbally or in writing.

\section{Results}

\section{Patient background characteristics}

The patient background characteristics are summarized in Table 2 . In regard to the gender distribution, the percentage of males was higher (about 70\%) in both groups, with no significant intergroup difference ( $\mathrm{P}=0.84$; Chi-squared test). There was also no significant difference between the two groups in terms of the age distribution $(\mathrm{P}=0.23$; Mann-Whitney $\mathrm{U}$ test). Cerebral palsy was the most common primary disorder in both groups, and the primary disorder pertained to organic disorders of the brain in all the patients. The onset of the disorders had been in the fetal or perinatal stage in all the patients. None of the patients had any concurrent disorders/complications that could potentially affect the oxidative stress levels, such as hypertension, diabetes mellitus, dyslipidemia, renal disease or infection. As for the AEDs that were prescribed, valproate sodium (VPA) was the most frequently prescribed, followed, in order, by phenytoin (PHT) and phenobarbital (PB). There were no significant intergroup differences in the type or number of AEDs prescribed, except that clonazepam was the most frequently prescribed drug among the newer AED group $(\mathrm{P}=$ 0.22; Mann-Whitney U test).

\section{Changes of the blood levels of d-ROMs and BAP and of the blood biochemical parameter values over time}

The blood levels of d-ROMs and BAP and the blood biochemical parameter values at measurement points 1 and 2 are shown 
Table 2. Background of the Patients

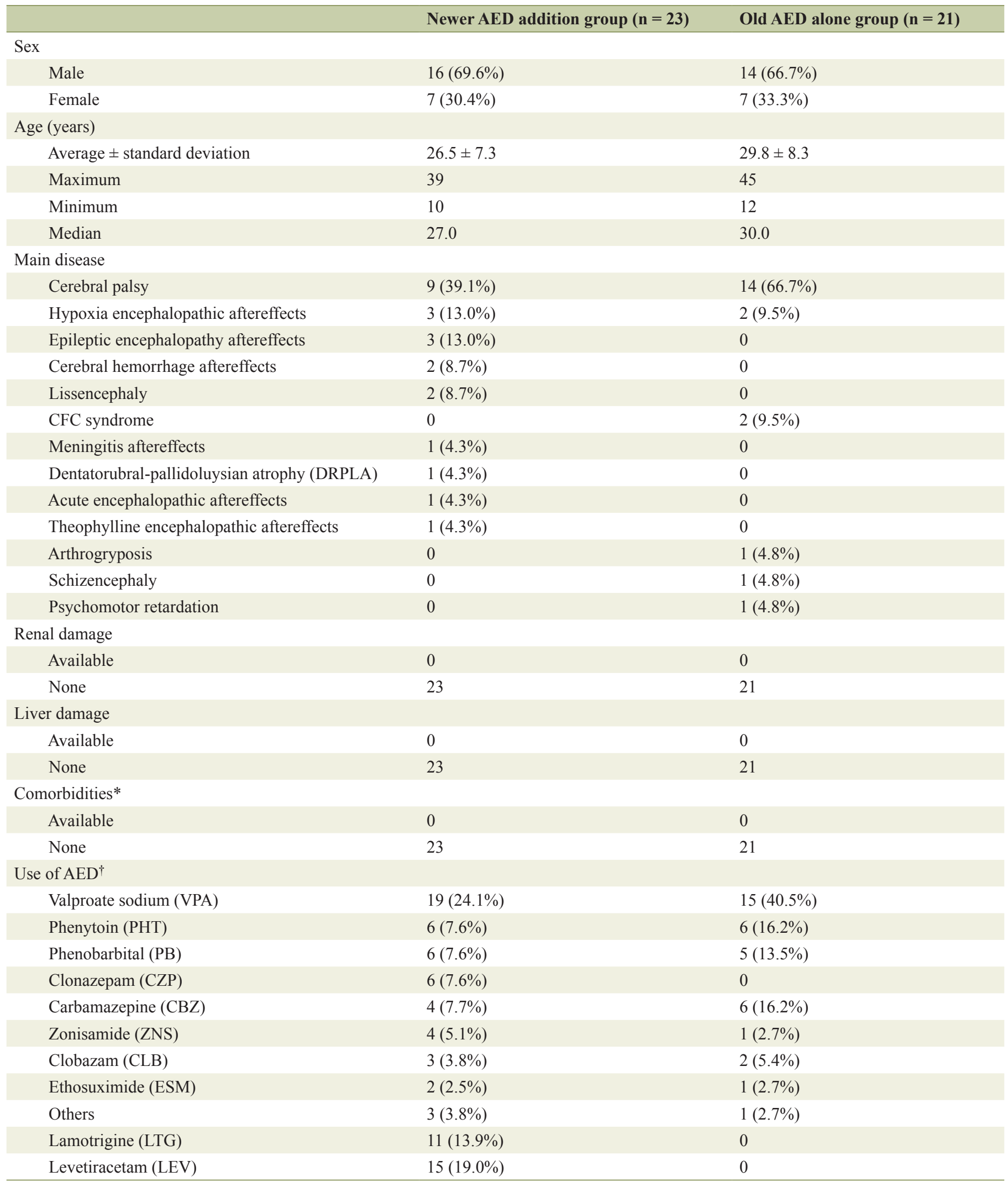

${ }^{*}$ Hypertension, diabetes, hyperlipidemia, kidney disease, infectious disease. ${ }^{\dagger}$ There is overlap in a value. 
Table 3. Blood Test Results (Newer AED Addition Group) $(n=23)$

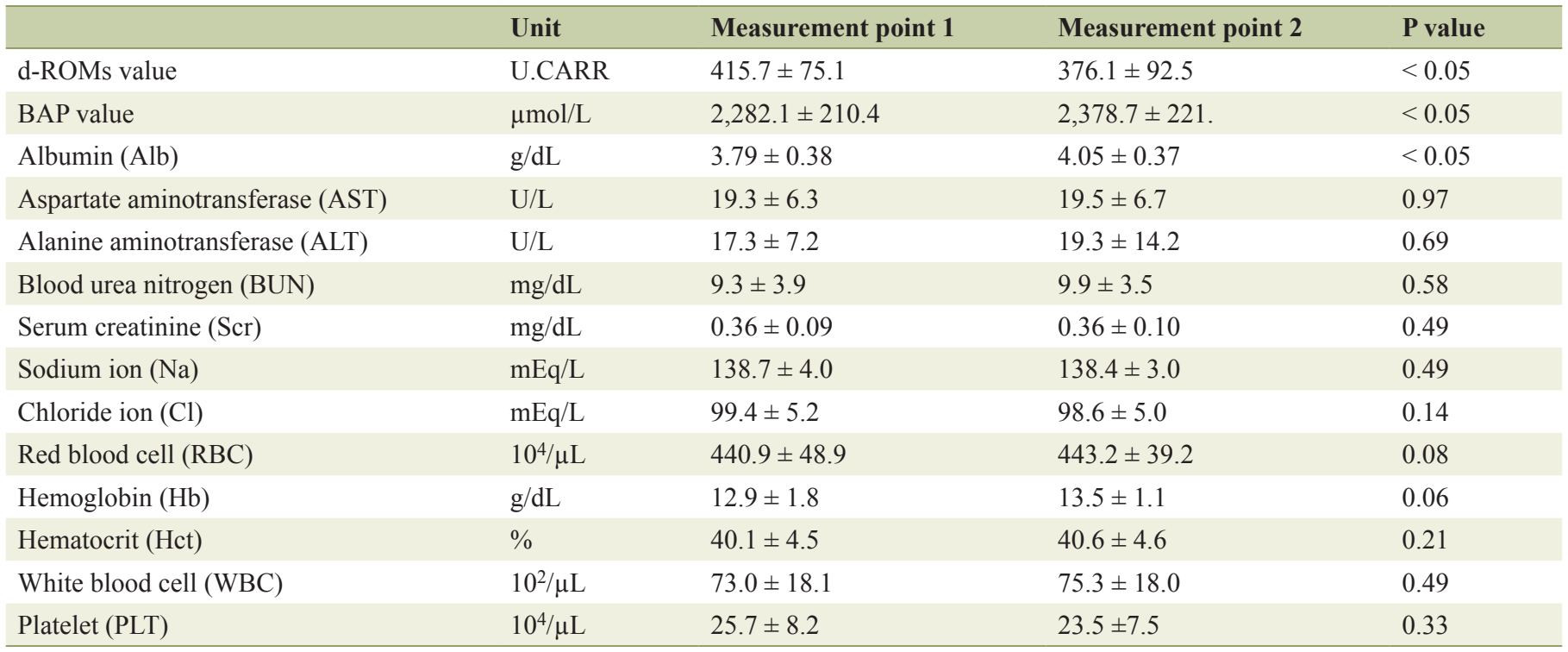

Measurement point 1: approximately 1 week ago when newer AED was added. Measurement point 2: it is approximately 1 year later from point 1. Wilcoxon signed-rank test was used for statistical analysis.

in Table 3 (newer AED group) and Table 4 (old AED group). The results demonstrated a significant decrease in the blood levels of d-ROMs and significant elevation of the BAP value at the second assay point in the newer AED group $(\mathrm{P}<0.05)$. A significant increase of the serum albumin level was also observed at the second measurement point in the newer AED group $(\mathrm{P}<0.05)$.

In the old AED group, significant increase of the blood levels of d-ROMs was observed at the second measurement point $(\mathrm{P}<0.05)$, whereas no significant changes were noted in the values of any of the other parameters measured.

\section{Relationship between the serum levels of albumin and cor- tisol}

A significant elevation of the serum albumin was observed at the second measurement point in the newer AED group. In view of reports purporting to demonstrate that the serum albumin level is affected by the serum cortisol, a well-known stress

Table 4. Blood Test Results (Old AED Addition Group) $(n=21)$

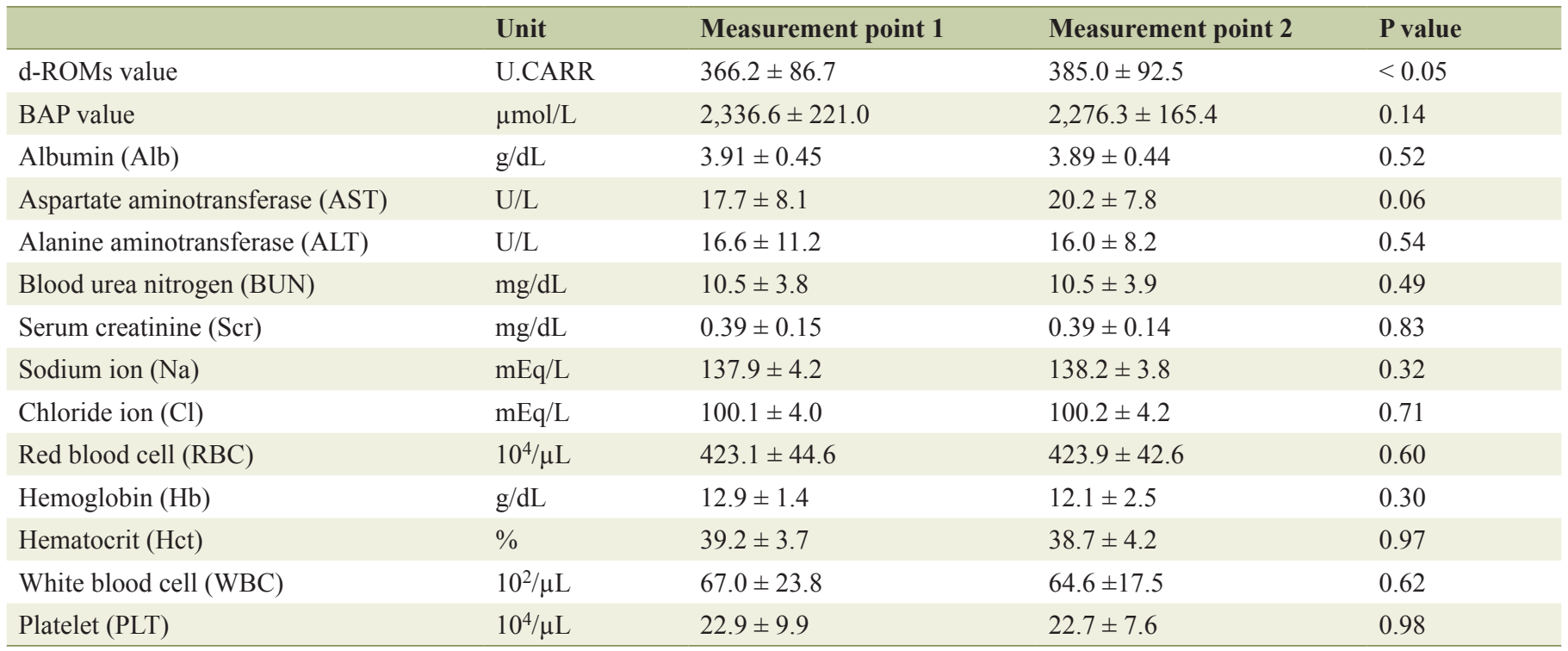

Measurement point 1: point of periodical drawing blood from April 2015 to September. Measurement point 2: it is approximately 1 year later from point 1. Wilcoxon signed-rank test was used for statistical analysis. 

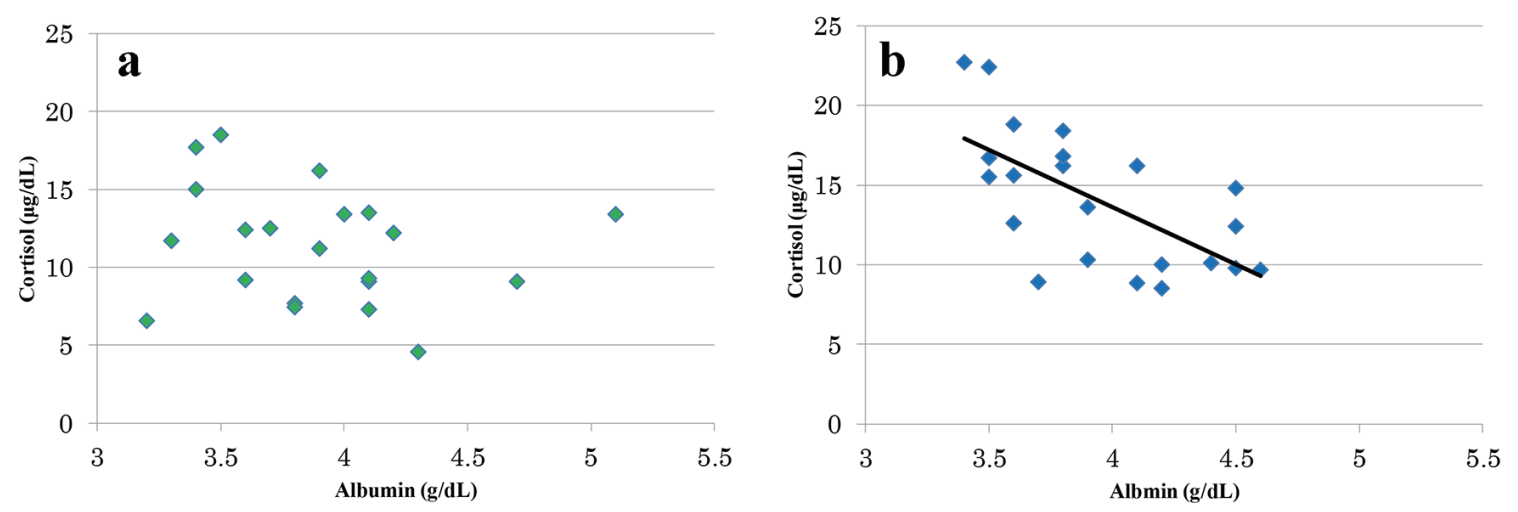

Figure 1. Relations of albumin level and the cortisol level. There was no significant correlation between albumin value and cortisol value in old AED group $(r=-0.195, P=0.39)(a)$. For the newer AED group, a significant correlation was found between the albumin value and the cortisol value $(r=-0.641, P<0.01)(b)$.

hormone $[8,9]$, we carried out an additional investigation to determine the relationship between the serum levels of albumin and cortisol. The relationship between the plasma cortisol level and serum albumin level measured at the second measurement point was determined. As the plasma cortisol levels show diurnal variations, the blood sample collection time for measurement of the plasma cortisol level was standardized to the morning time, before breakfast/morning doses of the AEDs (at $6 \mathrm{am}$ ), when the patient was in his/her usual stable state and not in a post-ictal phase or suffering/recovering from an infection.

Figure 1 illustrates the relationship between the serum levels of albumin and cortisol in the old AED group (Fig. 1a) and newer AED group (Fig. 1b), after excluding the data of one patient from the newer AED group in whom the relevant measurements proved infeasible because of an insufficient volume of the blood sample. As can be seen, there was no significant correlation between the two parameters in the old AED group, whereas a significant correlation was observed in the newer AED group $(\mathrm{r}=-0.641, \mathrm{P}<0.01)$.

\section{Discussion}

As for changes in the plasma levels of d-ROMs and plasma BAP value, these two parameters were found to show significant improvements at the second assay point in the newer AED group, whereas in the old AED group, the plasma d-ROM levels showed significant worsening. The drugs prescribed in the newer AED group, namely LTG and LEV, exert their actions via novel mechanisms, such as inhibition of glutamate release via blockade of $\mathrm{N}$ type $\mathrm{Ca}^{2+}$ channels (LTG) or action on synaptic vesicle protein SV2A (LEV). Addition of the newer AEDs with these novel mechanisms of action to the older AEDs which mainly exert their effects via $\mathrm{Na}^{+}, \mathrm{Ca}^{+}$channel inhibition, activation of the $\mathrm{Cl}^{-}$channel, activation of GABAergic neurons, etc., seems to afford synergic effects, leading to a reduction in the frequency of seizure and improvement in the oxidative stress level. Furthermore, LTG and LEV have been suggested to have cerebro- and nerve cell-protective effects [10-13], and LEV has been reported to inhibit induction of inflammatory cytokines such as interleukin $1-\beta$ and tumor necrosis factor- $\alpha(\mathrm{TNF}-\alpha)[14,15]$. It would be reasonable, therefore, to infer that these characteristics of the drugs accounted for the reduction in the oxidative stress level and improvement of the antioxidant potency in the newer AED group.

In the old AED group, there were a slight increase in frequency of seizure and a significant elevation of the plasma levels of d-ROMs, insomuch as these patients were maintained continuously on the conventional AEDs exerting their effects via the conventional mechanisms of action. It is thus inferred that the cerebral injuries caused by the repeated epileptic attacks, which sometimes even progressed to status epilepticus, and activation of the glial cells due to cerebral atrophy were the causes for the worsened plasma d-ROMs levels.

Age must also be taken into account as a factor affecting the level of oxidative stress. However, this factor was considered to have no appreciable impact in this study, in which there was no noticeable intergroup difference in the age distribution and the between measurement point interval was as short as about 1 year.

In the present study, significant elevation of the serum albumin was noted in the newer AED group at the second measurement point. Serum albumin is an important factor, in that it has been described to be correlated with the BAP [16], and it has been reported that the lower the serum albumin level, the higher the risk of death [17]. The serum albumin level is affected mainly by the balance among its absorption from the intestine, synthesis in the liver and excretion via the kidney. None of the patients in the present series of SMID patients had hepatic or renal impairment. Therefore, we reviewed the present data as reflecting the relationship between the intestinal absorption of albumin and the oxidative stress level.

Existence of an association has been reported between increased oxidative stress levels and sympathicotonia [18-20]. An increased risk of hypertension and some systemic effects emerge in the presence of enhanced sympathetic nervous activities. Intestines, also autonomically innervated, show depressed motility if the sympathetic nervous activities are enhanced, possibly affecting the absorption of dietary nutrients from the gut. Our preceding study demonstrated that the higher 


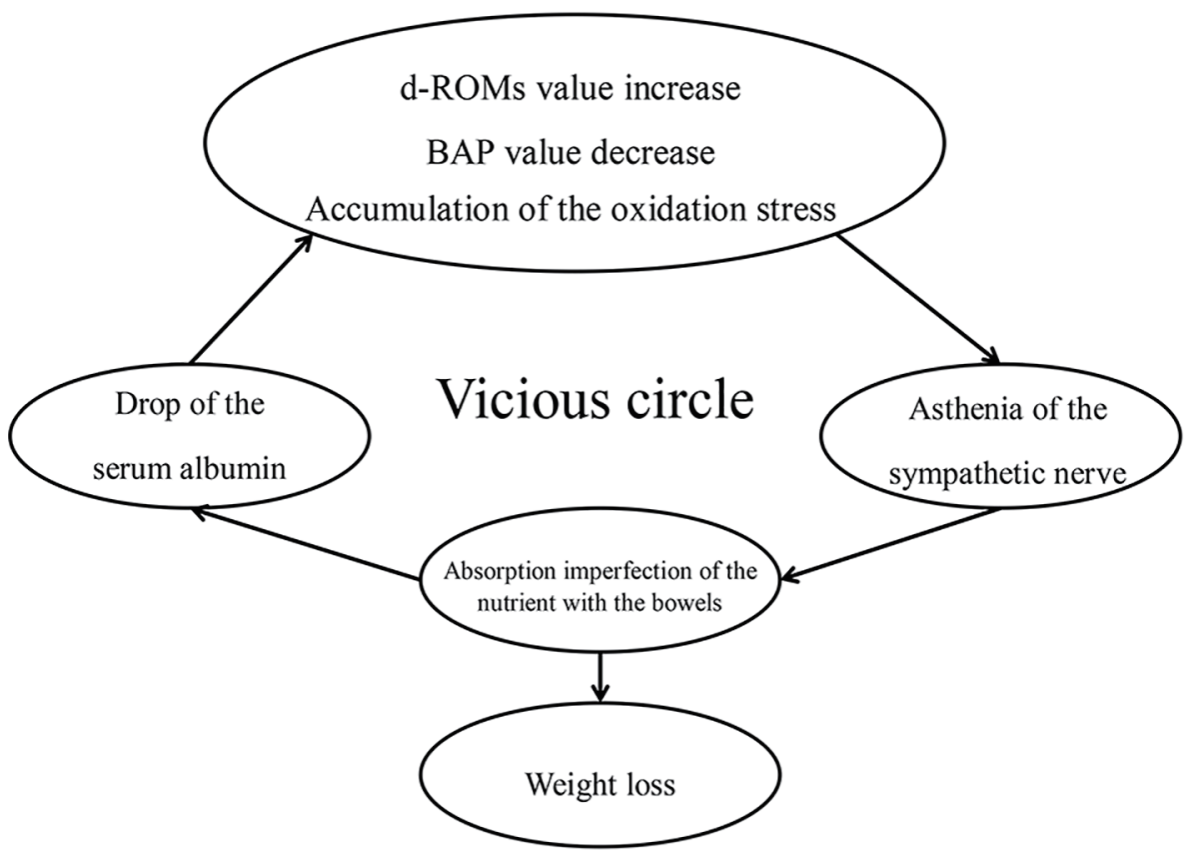

Figure 2. Vicious circle cycle by the oxidation stress.

the severity of SMID, the greater the number of AEDs prescribed and the number of patients with intractable epilepsy, and also that these patients tend to have a slender build [21]. It would follow that, as seen from Figure 2, once oxidative stress load occurs, the sympathetic nerves innervating the intestine become activated and the absorption of the antioxidant albumin diminishes, thereby resulting in a vicious cycle.
In the newer AED group, a significant decrease of the plasma levels of d-ROMs and an increase of the BAP were observed at the second measurement point. The present data did not reveal any evidence to suggest that the newer AEDs per se can cause improvement of the serum albumin level, even when compared with the conventional AEDs. As shown in Figure 3, nevertheless, it seems likely that the reduced oxidative stress

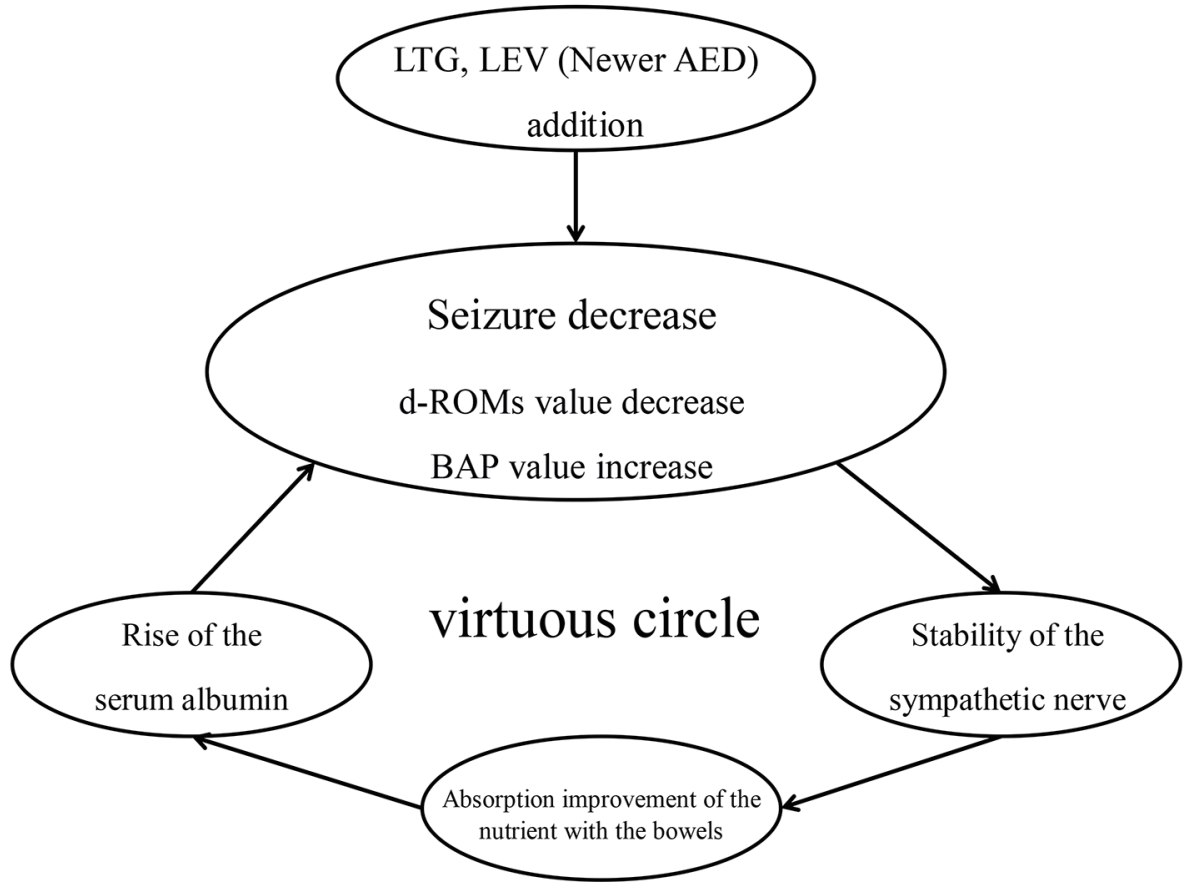

Figure 3. virtuous circle cycle by the oxidation stress decrease. 
level via suppression of seizure led as a virtuous cycle to the serum albumin elevation.

It is considered that in the presence of low albumin levels, the depressed capability to neutralize ROS and the reduced antioxidant potency may promote the development and further progression of intractable epilepsy and various disorders in SMID patients with epilepsy. From the present study data, the roles played by albumin are assumed to not only be confined to maintaining the plasma osmolality and nutrient and hormone transport, but also to reducing the cytotoxicity of oxidative stress in the disease state of epilepsy via autoxidation of albumin; hence, albumin plays a major role in maintaining homeostasis in the body.

The present study results also suggest that reduction in the oxidative stress load resulted in a decrease in the degree of autoxidation of albumin, thereby leading to elevation of the serum albumin level. It may be expected that maintained serum albumin levels improves patients' skinny build, energy metabolism and muscle strength, and contribute to more effective prevention of systemic disorders. In order to maintain stabilized oxidative stress levels in SMID patients with epilepsy, it may be important to not only make efforts to reduce the frequency of seizure, but also explore strategies and pharmacotherapeutic modalities that will enable maintenance of low levels of oxidation of albumin per se.

In regard to the relationship between the serum levels of albumin and cortisol, the present data demonstrated that the serum albumin level tended to increase (i.e., improved) and the plasma cortisol level tended to be reduced (i.e., improved) over time in the newer AED group, although patients showing abnormally low or abnormally high plasma levels of cortisol were infrequent among the present series. Cortisol secretion increases with sympathetic nervous activation, and excessive cortisol secretion results in a decrease of the serum albumin and diminution of the antioxidant potency, to cause accumulation of ROS. Serum cortisol is related to atherosclerosis, depressed immune functions, and decreased muscle synthesis, and cortisol is also more liable to be secreted under a chronic oxidative stress condition. It is thus considered important to prevent excessive cortisol secretion by reducing the oxidative stress and elevating the serum albumin level, as in the present study, to avoid propagation of vicious cycles.

Therefore, periodic measurements of oxidative stress markers such as d-ROMs and BAP, of the endogenous antioxidant albumin, and of the serum cortisol as a stress hormone may enable early detection of signs of changes in vivo of SMID patients who are started on the newer AEDs, and may be useful for prevention of sudden death and early detection of disorders.

The present study results revealed that treatment with the newer AEDs not only reduced the frequency of seizure in SMID patients, but also, as a secondary effect, improved the serum albumin level. The study also demonstrated that the serum albumin level is related to the serum level of the biologic stress hormone, cortisol.

\section{Conflict of Interest}

There is no conflict of interest to be disclosed.

\section{References}

1. Jones DP. Extracellular redox state: refining the definition of oxidative stress in aging. Rejuvenation Res. 2006;9(2):169-181.

2. Yoshikawa T. Free radicals and medicine. Journal of Kyoto Prefectural University of Medicine. 2011;120(6):381391.

3. Morimoto M, Satomura S, Hashimoto T, Ito E, Kyotani S. Oxidative Stress Measurement and Prediction of Epileptic Seizure in Children and Adults With Severe Motor and Intellectual Disabilities. J Clin Med Res. 2016;8(6):437444.

4. Yoshida R, Ishizaki A, Sato J, Fukuyama Y. [A clinical study of sudden death in the severely handicapped persons]. No To Hattatsu. 1995;27(6):466-472.

5. Ishizaki A, Shinozaki M, Yoshida R, Kurata K, Sakamoto K, Shinohara T, Sasaki H, et al. [A clinical study of death in profound mental retardation with motor disturbance]. No To Hattatsu. 1990;22(4):357-363.

6. Anraku M. [Elucidation of the mechanism responsible for the oxidation of serum albumin and its application in treating oxidative stress-related diseases]. Yakugaku Zasshi. 2014;134(9):973-979.

7. Karceski S, Morrell MJ, Carpenter D. Treatment of epilepsy in adults: expert opinion, 2005. Epilepsy Behav. 2005;7(Suppl 1):S1-64; quiz S65-67.

8. Hamrahian AH, Oseni TS, Arafah BM. Measurements of serum free cortisol in critically ill patients. N Engl J Med. 2004;350(16):1629-1638.

9. Ho JT, Ai-Musalhi H, Chapman J, Quach T, Tomas PD, Baqley CJ, Lewis JG, et al. Septic shock and sepsis: A comparison of total and free plasma cortisol level. J Clin Endcrinol Metab. 2006;91(1):105-114.

10. Kim YJ, Ko HH, Han ES, Lee CS. Lamotrigine inhibition of rotenone- or 1-methyl-4-phenylpyridinium-induced mitochondrial damage and cell death. Brain Res Bull. 2007;71(6):633-640.

11. Smith SE, Meldrum BS. Cerebroprotective effect of lamotrigine after focal ischemia in rats. Stroke. 1995;26(1):117-121; discussion 121-112.

12. Gibbs JE, Walker MC, Cock HR. Levetiracetam: antiepileptic properties and protective effects on mitochondrial dysfunction in experimental status epilepticus. Epilepsia. 2006;47(3):469-478.

13. Belcastro V, Pierguidi L, Tambasco N. Levetiracetam in brain ischemia: clinical implications in neuroprotection and prevention of post-stroke epilepsy. Brain Dev. 2011;33(4):289-293.

14. Marini H, Costa C, Passaniti M, Esposito M, Campo GM, Ientile R, Adamo EB, et al. Levetiracetam protects against kainic acid-induced toxicity. Life Sci. 2004;74(10):12531264.

15. Stettner M, Dehmel T, Mausberg AK, Kohne A, Rose CR, Kieseier BC. Levetiracetam exhibits protective properties on rat Schwann cells in vitro. J Peripher Nerv Syst. 2011;16(3):250-260.

16. Shimo T, Takahasi M, Takewa R, Tanaka S, Hasui M, 
Kaneko K. Role of serum albumin as an antioxidant in idiopathic nephrotic syndrome. Japanese Journal of Pediatric Nephrology. 2010;23(2):164-167.

17. Kaneko K, Kimata T, Tsuji S, Shimo T, Takahashi M, Tanaka S. Serum albumin level accurately reflects antioxidant potentials in idiopathic nephrotic syndrome. Clin Exp Nephrol. 2012;16(3):411-414.

18. Kishi T. Regulation of the sympathetic nervous system by nitric oxide and oxidative stress in the rostral ventrolateral medulla: 2012 Academic Conference Award from the Japanese Society of Hypertension. Hypertens Res. 2013;36(10):845-851.

19. Hakucho A, Liu J, Liu X, Fujimiya T. Carvedilol improves ethanol-induced liver injury via modifying the interaction between oxidative stress and sympathetic hyperactivity in rats. Hepatol Res. 2014;44(5):560-570.

20. Fujima M, Kuwaki T, Ando K, Fujita T. Sympatho-inhibitory action of endogenous adrenomedullin through inhibition of oxidative stress in the brain. Hypertention. 2005;45(6):1165-1172.

21. Morimoto M, Hashimoto T, Shigeko S, Shimakawa $\mathrm{S}$, Nakatsu T, Kyotani S, et al. Investigation of factors contributing to the current status of patients with severe motor and intellectual disabilities and the relationships between those factors. Journal of Severe Motor and Intellectual Disabilities. 2014;39(3):387-395. 